\title{
Implementation of personalised medicine policies in mental healthcare: results from a stated preference study in the UK
}

\author{
Alan J. McMichael*, Joseph P. M. Kane*, Jonathan J. Rolison, Francis A. O’Neill, Marco Boeri† and \\ Frank Kee†
}

\section{Background}

Public support for the implementation of personalised medicine policies (PMPs) within routine care is important owing to the high financial costs involved and the potential for redirection of resources from other services.

\section{Aims}

We aimed to determine the attributes of a PMP most likely to elicit public support for implementation. We also aimed to determine whether such support differed between a depression PMP and one for cystic fibrosis.

\section{Method}

In a discrete-choice experiment, paired vignettes illustrating both the current model of care $(\mathrm{CMOC})$ and a hypothetical PMP for either depression or cystic fibrosis were presented to a representative sample of the UK public $(n=2804)$. Each vignette integrated varying attributes, including anticipated therapeutic benefit over $\mathrm{CMOC}$, and the annual cost to the taxpayer. Respondents were invited to express their preference for either the PMP or CMoC within each pair.

\section{Results}

The financial cost was the most important attribute influencing public support for PMPs. Respondents favoured PMP implementation where it benefited a higher proportion of patients or was anticipated to be more effective than $\mathrm{CMOC}$. A reduction in services for non-eligible patients reduced the likelihood of support for PMPs. Respondents were more willing to fund PMPs for cystic fibrosis than for depression.

\section{Conclusions}

Cost is a significant factor in the public's support for PMPs, but essential caveats, such as protection for services available to PMP-ineligible patients, may also apply. Further research should explore the factors contributing to condition-specific nuances in public support for PMPs.

\section{Keywords}

Economics; public health; personalised medicine; discretechoice experiments; public preference.

\section{Copyright and usage}

(c) The Author(s), 2022. Published by Cambridge University Press on behalf of the Royal College of Psychiatrists. This is an Open Access article, distributed under the terms of the Creative Commons Attribution-NonCommercial-NoDerivatives licence (https://creativecommons.org/licenses/by-nc-nd/4.0/), which permits non-commercial re-use, distribution, and reproduction in any medium, provided the original work is unaltered and is properly cited. The written permission of Cambridge University Press must be obtained for commercial re-use or in order to create a derivative work.
The use of biomarkers to tailor treatments to a subgroup of patients, known as personalised medicine or stratified medicine, ${ }^{1}$ may represent a more precise and potentially effective approach to managing mental disorders. ${ }^{2}$ However, the financial cost and reorganisation necessary for implementation of this approach represent significant barriers to its adoption. ${ }^{3}$

Through taxation, the population of most countries are primary stakeholders in their healthcare systems and act as drivers of change by electing politicians whose healthcare agendas resonate with their own. Surveys suggest that the public welcomes targeted and more effective treatments, but that enthusiasm for personalised medicine policies (PMPs) may be tempered by concerns over consequential restrictions on the care of other patients. ${ }^{4}$

Although psychiatric disorders contribute $22.8 \%$ of the total global disease burden, ${ }^{5}$ the UK spends $10.8 \%$ of its annual health expenditure on mental illness. ${ }^{6}$ A PMP would require an even greater level of resource redistribution, with consequences for other services. Determining PMPs' acceptability in mental health settings is, therefore, an essential step towards implementation.

Using a discrete-choice experiment (DCE) approach, this study aims to evaluate the degree to which the public is willing (in a hypothetical referendum) to support a PMP over current models of care

\footnotetext{
* Joint first authors

† Joint senior authors
}

(CMoC). It also aims to determine the characteristics of the PMPs that would have broader support. It does this by comparing two contrasting exemplar PMPs, one aimed at treating depression and another aimed at treating cystic fibrosis, for which a PMP is already in routine use.

\section{Method}

\section{Materials}

We used a DCE approach, in which respondents were presented with pairs of vignettes, determined by an experimental design, and in each case asked to choose the vignette they preferred. Each vignette was defined by varying values for a consistent set of attributes. ${ }^{7}$ Respondents' choices depended on the perceived importance of the attribute levels. The implied importance weights of the attributes and attribute levels could be estimated from the observed patterns of choices through statistical analysis (as described below). ${ }^{8}$

In this study, respondents were asked to decide whether they would favour implementing a hypothetical PMP over CMoC for the treatment of either depression or cystic fibrosis.

We chose cystic fibrosis, an autosomal disorder affecting one in 2500 people, as a comparator to depression, as it is a condition for which personalised medicine models of treatment are already available. The $5 \%$ of patients with cystic fibrosis with specific mutations 
in the cystic fibrosis transmembrane conductance regulator (CFTR) gene are eligible for ivacaftor therapy, a treatment costing approximately $£ 182000$ per patient-year. ${ }^{9}$

Four attributes of the proposed PMP characterised each vignette.

(a) The proportion of patients who would receive PMP treatment $(10 \%, 30 \%, 50 \%, 70 \%$ or $90 \%)$.

(b) The potential effectiveness of the PMP treatment (10\%, 30\% or $50 \%$ more effective than $\mathrm{CMoC}$ ).

(c) Whether or not supportive services available to all patients, caregivers and their families would be reduced.

(d) The additional cost in UK sterling to each taxpayer were the PMP to be implemented ( $£ 10, £ 20, £ 40, £ 70$ or $£ 90$ ).

The CMoC did not vary across vignettes; all patients would continue to receive the same standard treatment, and all patients, their caregivers and their families would continue to receive the current level of supportive services.

To select the attributes to be assessed in the DCE, the study team reviewed the literature on preferences for stratified medicine at the beginning of the study. Levels for each clinical attribute were determined based on evidence from clinical studies available at the time of the study design. To elicit respondents' willingness to pay (WTP), the DCE included a cost attribute as an additional annual tax to each taxpayer. The cost of the current (general) healthcare system was set to $£ 0$.

The depression and cystic fibrosis vignettes were identical except for the medical condition to which they referred. Personalised medicine was referred to as 'stratified medicine' throughout the vignettes used.

Each vignette was presented to the respondent within the following scenario:

'Imagine that a referendum is being conducted to decide on the matter of implementing a proposed new health care system to include stratified (personalised) medicine for cystic fibrosis. If there is a majority of "no" votes, the programme would not be implemented and only the current (general) health care system would be maintained. If there is a majority of "yes" votes, the programme would be implemented, and would be funded through an annual tax paid by all households in the UK'.

Respondents were then presented with vignettes, in the format shown in Fig. 1, and were asked: 'Would you vote in favour of the proposed new health care system to include stratified medicine for this condition?'

A full factorial experimental design, including all possible combinations of the attribute levels, would have required 600 unique vignettes. To reduce the number of vignettes to a more feasible level for respondents to complete within a single session, ${ }^{7}$ a D-efficient experimental design was generated using Ngene

\begin{tabular}{|c|c|c|}
\hline & $\begin{array}{l}\text { Current (general) } \\
\text { healthcare system }\end{array}$ & $\begin{array}{l}\text { Proposed new healthcare system } \\
\text { to include stratified medicine }\end{array}$ \\
\hline $\begin{array}{l}\text { Proportion of patients } \\
\text { with cystic fibrosis who } \\
\text { will receive the stratified } \\
\text { medicine treatment }\end{array}$ & $\begin{array}{l}\text { All patients with cystic fibrosis } \\
\text { receive the standard treatments as } \\
\text { part of their general healthcare }\end{array}$ & $\begin{array}{l}30 \% \text { of patients with cystic fibrosis } \\
\text { will receive the stratified medicine } \\
\text { treatment. All other patients } \\
\text { with cystic fibrosis will receive the } \\
\text { standard treatment }\end{array}$ \\
\hline $\begin{array}{l}\text { Potential effectiveness } \\
\text { of the stratified medicine } \\
\text { treatment }\end{array}$ & $\begin{array}{l}\text { Only the standard treatment } \\
\text { is available }\end{array}$ & $\begin{array}{l}\text { The stratified medicine treatment } \\
\text { is } 50 \% \text { more effective on average } \\
\text { than the standard treatment }\end{array}$ \\
\hline $\begin{array}{l}\text { Supportive service } \\
\text { available to all patients } \\
\text { with cystic fibrosis, carers } \\
\text { and their families in addition } \\
\text { to medical treatment }\end{array}$ & $\begin{array}{l}\text { All patients with cystic fibrosis, } \\
\text { carers and their families receive } \\
\text { the current level of services in } \\
\text { addition to medical treatment }\end{array}$ & $\begin{array}{l}\text { Supportive services available to all } \\
\text { patients with cystic fibrosis, carers } \\
\text { and their families will be reduced }\end{array}$ \\
\hline $\begin{array}{l}\text { Additional cost to each } \\
\text { taxpayer }\end{array}$ & No additional cost to taxpayers & $\begin{array}{c}\text { Additional } £ 100 \text { paid annually by } \\
\text { each taxpayer }\end{array}$ \\
\hline \multicolumn{3}{|c|}{$\begin{array}{l}\text { Would you vote in favour of the proposed new healthcare system to include } \\
\text { stratified medicine for cystic fibrosis? }\end{array}$} \\
\hline
\end{tabular}

Fig. 1 Example of a cystic fibrosis vignette. Based on the proportion of patients eligible for the PMP, its potential effectiveness, whether there would be a reduction in non-medical services, and the associated annual tax increase, respondents indicated whether they would vote in favour of the proposed personalised medicine policy. 'Cystic fibrosis' was replaced with 'depression' in the depression vignettes. 
(ChoiceMetrics, 2014). In the D-efficient design, a regression model is pre-specified with prior expectations about each attribute coefficient. Starting from the full factorial design, the D-efficient algorithm creates fractional factorial designs by minimising an index based on the variance-covariance matrix for the pre-specified regression model.

\section{Pre-test}

Efficient experimental design requires some prior knowledge of the preferences to be elicited (as these enter the variance-covariance matrix). Thus, in line with good DCE practice ${ }^{7}$ and past research, ${ }^{10,11}$ we conducted a pre-test with 100 respondents based on an orthogonal design with information on preference direction (e.g. to avoid dominated pairs in the design) and used the data to gain priors to construct a D-efficient design. This pre-test helped to ensure that the vignettes and attributes were designed in a way that was easy for the study participants to understand. Feedback from the pre-test study was positive, with participants in the convenience sample indicating that the study was straightforward and that the attributes and levels were realistic. After the pre-test, we generated 48 unique vignettes; these were divided into four blocks, each containing 12 vignettes. Each respondent was randomly assigned to one of the four blocks. The order of vignettes was randomised for each respondent.

\section{Respondents}

We employed a private survey company (formerly known as Survey Sampling International, now Dynata) to recruit a sample of respondents from across the UK. Dynata provided access to a sample representative of the population in terms of age, gender and socioeconomic status. Respondents $(N=2804)$ were randomly assigned to receive either the depression $(N=1360)$ or cystic fibrosis $(N=1444)$ vignettes.

Two screening questions were used to assess whether respondents currently paid tax, had previously been treated for depression, knew someone who had been treated for depression, had been treated for cystic fibrosis or knew someone who had been treated for cystic fibrosis. The vignettes were presented in a randomly generated order for each respondent. If a respondent had been treated for cystic fibrosis or knew someone who had been treated for cystic fibrosis, they were automatically assigned to the cystic fibrosis vignettes (rather than the depression vignettes), owing to cystic fibrosis being less prevalent than depression.

\section{Statistical analysis}

Discrete-choice questions generate cross-sectional or time-series data that require analysis using multinomial and mixed logistic regression. ${ }^{8,12}$ Data were first explored with a conditional-multinomial logit (MNL) model as a benchmark to define model specification without accommodating preference heterogeneity, However, as widely acknowledged in the stated preference literature, unobserved variation in preferences across the sample can bias estimates obtained with MNL models. Therefore, different mixed logit models assuming different specifications were explored (i.e., continuous and discrete distributions to represent preference heterogeneity as well as uncorrelated and correlated random parameters). The final model specification, presented in this paper, was a random-parameters logit (RPL, which assumes continuous distributions to represent preference heterogeneity across the sample) model with full covariance among coefficients, accounting for the cross-section/panel nature of the data and allowing for unexplained preference heterogeneity, as well as all sources of correlation (such as that induced by scale heterogeneity). ${ }^{13}$ The parameters for each attribute (the proportion of patients expected to benefit, the increased effectiveness of PMP, the reduction in supportive services and the annual tax increase) were included as predictors in the regression model on respondents' choices and assumed to have a normal distribution. As the RPL model has no closed form, ${ }^{8}$ the models were estimated using maximum simulated likelihood with 500 Halton draws. In each model specification, a dummy coded variable of 1 (for respondents who were asked to think about depression) or 0 (for respondents who were asked to think about cystic fibrosis) was interacted with all the model parameters to test for systematic differences in preferences between each subgroup. A Wald test of the joint significance of the interaction terms indicated whether preferences between the two groups were statistically systematically different.

The conditional relative attribute importance (CRAI) was computed by considering the levels included for the attribute and the preference. The CRAI is the importance of each attribute relative to all other attributes included in the study, conditional on the range of levels of the attribute. The CRAI can be interpreted as the maximum change in utility achievable with any attribute, relative to all others, given the levels chosen for the attributes in the study. The CRAI was standardised considering the total change in utility achievable, moving from the least to the most preferred level for all attributes (the sum of the conditional relative importance of each attribute), equal to 100, and each CRAI was rescaled accordingly. In this way, the CRAI of each attribute could also be interpreted as a percentage change of the total utility change achievable. The standard errors and the $95 \%$ confidence intervals for these differences were calculated using the delta method.

Finally, WTP for each attribute (as part of a tax increase) was calculated as the negative ratio of the coefficient of any attribute to the coefficient of the tax increase. For example, the increase in the amount that respondents would be willing to pay in tax when $10 \%$ of patients would benefit was estimated as the negative $\beta$ (proportion of patients benefiting) divided by $\beta$ (tax increase). The WTP reflects the change in welfare given by any unit change in each benefit. In other words, WTP is the amount of money that respondents are willing to give up for an increase in any specific benefit. All statistical analyses were conducted in STATA version 16 (StataCorp, 2019) with the mixlogit command. ${ }^{14}$

\section{Ethics statement}

The authors assert that all procedures contributing to this work comply with the ethical standards of the relevant national and institutional committees on human experimentation and with the Helsinki Declaration of 1975, as revised in 2008. All procedures involving human subjects/patients were approved by Queens University Belfast Ethics Committee. Subjects provided written consent to participate in the study.

\section{Consent statement}

All survey respondents provided electronic informed consent before starting the survey.

\section{Results}

\section{Recruiting and demographic characteristics}

The survey link was accessed by 3105 potential respondents. Of these, 301 did not complete any of the DCE questions and could not be included in the analysis. Demographic information for the remaining 2804 respondents is provided in Table 1. The median and mean times for each participant to complete the survey were approximately $5 \mathrm{~min}$. No respondents were excluded for completing the survey too quickly, and when 41 subjects who completed the 


\begin{tabular}{|c|c|c|}
\hline \multicolumn{3}{|l|}{ Variable } \\
\hline & Mean & Range \\
\hline Age (s.d.), years & $44(16.75)$ & $16-75$ \\
\hline Gender & $n$ & $\%$ \\
\hline Male & 1416 & 49.5 \\
\hline Female & 1388 & 49.5 \\
\hline \multicolumn{3}{|l|}{ Highest education } \\
\hline Primary school only & 52 & 1.9 \\
\hline GCSE & 595 & 21.2 \\
\hline A-level & 465 & 16.5 \\
\hline College (e.g. NVQ) & 611 & 21.8 \\
\hline University or higher & 1081 & 38.6 \\
\hline \multicolumn{3}{|l|}{ Employment status } \\
\hline Working full time & 1390 & 49.6 \\
\hline Working part time & 497 & 17.7 \\
\hline Unemployed & 48 & 1.7 \\
\hline Student & 269 & 9.6 \\
\hline Retired & 536 & 19.1 \\
\hline Homemaker & 64 & 2.2 \\
\hline \multicolumn{3}{|l|}{ Income before tax } \\
\hline Less than $£ 10000$ & 297 & 10.6 \\
\hline$£ 10000$ to $£ 30000$ & 1244 & 44.4 \\
\hline$£ 30001$ to $£ 50000$ & 800 & 28.6 \\
\hline More than $£ 50000$ & 463 & 16.5 \\
\hline \multicolumn{3}{|c|}{ Have you ever been treated for depression? } \\
\hline Yes & 719 & 25.6 \\
\hline No & 2085 & 74.3 \\
\hline \multicolumn{3}{|c|}{ Do you know anyone who has been treated for depression? } \\
\hline Yes & 1792 & 64 \\
\hline No & 1012 & 36 \\
\hline \multicolumn{3}{|c|}{ Have you ever been treated for cystic fibrosis? } \\
\hline Yes & 20 & 0.7 \\
\hline No & 2784 & 99.3 \\
\hline \multicolumn{3}{|c|}{ Do you know anyone who has been treated for cystic fibrosis? } \\
\hline Yes & 337 & 12 \\
\hline No & 2467 & 88 \\
\hline \multicolumn{3}{|c|}{ People in household excluding you } \\
\hline 0 & 370 & 13.2 \\
\hline 1 & 773 & 27.6 \\
\hline 2 & 702 & 25 \\
\hline 3 & 500 & 17.8 \\
\hline 4 & 309 & 11 \\
\hline 5 or more & 150 & 5.4 \\
\hline \multicolumn{3}{|c|}{ Children below 16 in household } \\
\hline 0 & 1945 & 69.4 \\
\hline 1 & 439 & 15.7 \\
\hline 2 & 313 & 11.2 \\
\hline 3 & 76 & 2.7 \\
\hline 4 & 25 & 0.9 \\
\hline 5 or more & 6 & 0.21 \\
\hline \multicolumn{3}{|c|}{ What is your marital status? } \\
\hline Single (never married) & 917 & 32.7 \\
\hline Married & 1228 & 43.8 \\
\hline Living as married & 341 & 12.2 \\
\hline Divorced & 188 & 6.7 \\
\hline Separated & 53 & 1.9 \\
\hline Widowed & 77 & 2.8 \\
\hline
\end{tabular}

survey in under 2 min were excluded from analysis, we observed no significant changes to the results produced. We identified 357 respondents who always chose in favour of implementing the PMP ( $n=208$ in the cystic fibrosis sample and $n=149$ in the depression sample), and 459 respondents who always selected the CMOC ( $n=224$ in the cystic fibrosis sample and $n=235$ in the depression sample). These respondents were included in the model presented in Table 2 and reflected in the alternative specific constant $(\mathrm{CMoC})$.

Respondents' mean age was 44 years (s.d. $=16.8$; range, $16-75$ years), similar to that reported by the 2011 Census ( 40 years), ${ }^{15}$ and $50 \%$ of respondents were male. A total of $39 \%$ of respondents indicated that they had completed a university degree or higher: $21 \%$ had at least General Certificate of Secondary Education (GCSE)-level education, and $16.5 \%$ had completed higher secondary education ('A-levels'). The sample therefore included slightly more highly educated respondents than the general population, of whom $27 \%$ possess a university degree or higher. ${ }^{15}$ Just under half $(49.5 \% ; 1390 / 2804)$ of respondents reported being employed full time, slightly less than in the 2011 Census population, in which $55 \%$ were employed full time. ${ }^{15}$ In the sample, 719 (25.6\%) respondents had been treated for depression, and 1792 respondents (63.9\%) knew someone who had been treated for depression. Twenty respondents $(0.7 \%)$ had been treated for cystic fibrosis and 337 (12.0\%) knew someone who had been treated for cystic fibrosis.

\section{Respondents' evaluation of PMP}

Respondents were significantly more likely to vote in favour of the PMP than CMoC (indicated by the negative coefficient for the $\mathrm{CMoC}$ parameter). Respondents preferred a PMP for which a higher proportion of patients would be eligible and a higher effectiveness of treatment was anticipated (Table 2). Conversely, respondents were significantly less likely to vote in favour of a PMP if it implied a reduction in supportive services or an annual tax increase. Interestingly, respondents did not have statistically different preferences for each attribute on the basis of condition (cystic fibrosis or depression) but were more likely to favour a $\mathrm{CMoC}$ for depression than one for cystic fibrosis. The variance-covariance matrix (Table 2) demonstrates that the standard deviations of the normal distributions assumed for each parameter (those on the main diagonal of the matrix) were statistically significantly different from zero, and that distributions were correlated (indicating the presence of correlation that may have been induced by scale heterogeneity). ${ }^{12,13}$

\section{CRAl and WTP estimates}

The estimates presented in Table 2 were used to calculate the CRAI and WTP of the treatment attributes in the study (Table 3). Over the ranges presented in the survey, reducing the tax contribution from $£ 90$ to $£ 10$ was the most important attribute (accounting for over $50 \%$ of the total utility gain achievable), followed by the proportion of patients benefiting from the treatment increasing from $10 \%$ to $90 \%$. As expected from the model estimates presented in Table 2, the CRAI of each attribute was similar for respondents given cystic fibrosis vignettes and those given ones for depression.

The WTP estimates, presented in the last three columns of Table 3, demonstrate that respondents were willing to pay almost $£ 17$ to avoid a reduction in supportive services. They were also prepared to pay approximately $£ 0.50$ per percentage point increase in the proportion of patients that stood to benefit and the effectiveness of PMP. Respondents were therefore willing to pay approximately $£ 5$ for a 10-percentage-point increase either in the proportion of patients benefiting from the treatment or in the effectiveness. Interestingly, all else being equal, respondents were willing to pay to maintain CMoCs for depression (£4.05) but would require compensation to maintain support for $\mathrm{CMoCs}$ for cystic fibrosis $(-£ 3.62)$, which could be interpreted as a positive WTP to implement a PMP for cystic fibrosis.

\section{Discussion}

The financial cost involved in implementing emerging PMPs may represent a significant barrier to their adoption in mental health 
Table 2 Random parameter model on whether respondents were in favour of implementing the personalised medicine policy $(N=2804)$

\begin{tabular}{|c|c|c|c|c|c|}
\hline & \multirow[b]{2}{*}{ Coefficient } & \multirow[b]{2}{*}{ s.e. } & \multicolumn{3}{|c|}{$95 \% \mathrm{Cl}$} \\
\hline & & & LB & UB & \\
\hline \multicolumn{6}{|l|}{ Cystic fibrosis } \\
\hline Proportion of patients benefiting & $0.025^{*}$ & 0.002 & 0.021 & 0.028 & \\
\hline Increased effectiveness & $0.031^{*}$ & 0.003 & 0.026 & 0.036 & \\
\hline Supportive services reduced & $-0.498 *$ & 0.040 & -0.576 & -0.420 & \\
\hline Tax increase & $-0.060^{*}$ & 0.002 & -0.064 & -0.055 & \\
\hline $\mathrm{CMOC}$ & $-0.215^{\star}$ & 0.146 & -0.501 & 0.070 & \\
\hline \multicolumn{6}{|l|}{ Delta depression (interacted parameter) } \\
\hline Proportion of patients benefitting & 0.004 & 0.002 & -0.001 & 0.009 & \\
\hline Increased effectiveness & -0.004 & 0.004 & --0.011 & 0.003 & \\
\hline Supportive services reduced & -0.048 & 0.056 & -0.158 & 0.062 & \\
\hline Tax increase & -0.003 & 0.003 & -0.009 & 0.003 & \\
\hline $\mathrm{CMOC}$ & $0.456^{*}$ & 0.204 & 0.056 & 0.857 & \\
\hline \multicolumn{6}{|l|}{ Variance-covariance matrix } \\
\hline Proportion of patients benefiting & $\begin{array}{l}\text { Proportion of patients benefiting } \\
0.001^{*}\end{array}$ & Increased effectiveness & Supportive services reduced & Tax increase & $\mathrm{CMOC}$ \\
\hline Increased effectiveness & $0.001^{*}$ & $0.001^{*}$ & & & \\
\hline Supportive services reduced & $0.005^{*}$ & $0.007^{*}$ & $0.634^{*}$ & & \\
\hline Tax increase & $0.001 *$ & $0.001^{*}$ & 0.002 & $0.003^{*}$ & \\
\hline $\mathrm{CMOC}$ & $0.076^{*}$ & $0.082^{*}$ & $0.804 *$ & 0.015 & 17.508 \\
\hline Null log-likelihood & -23323.016 & & & & \\
\hline Log-likelihood & -14680.405 & & & & \\
\hline K & 25 & & & & \\
\hline Pseudo R2 & 0.369 & & & & \\
\hline $\mathrm{BIC}$ & $29621 / 403$ & & & & \\
\hline
\end{tabular}

Table 3 Conditional relative attribute importance (CRAI) and willingness to pay (WTP) for each attribute

\begin{tabular}{|c|c|c|c|c|c|c|}
\hline & \multirow[b]{2}{*}{ CRAI } & \multicolumn{2}{|c|}{$95 \% \mathrm{Cl}$} & \multirow[b]{2}{*}{ WTP } & \multicolumn{2}{|c|}{$95 \% \mathrm{Cl}$} \\
\hline & & LB & UB & & LB & UB \\
\hline \multicolumn{7}{|l|}{ Cystic fibrosis } \\
\hline Proportion of patients benefiting & 20.54 & 17.70 & 23.38 & 0.41 & 0.36 & 0.47 \\
\hline Increased effectiveness & 12.91 & 10.82 & 15.01 & 0.52 & 0.43 & 0.61 \\
\hline Supportive services reduced & 10.44 & 8.79 & 12.08 & 16.7 & 13.94 & 19.53 \\
\hline Tax increase & 56.11 & 51.63 & 60.60 & & & \\
\hline $\begin{array}{l}\text { Current model of care } \\
\text { Delta depression (interacted paran }\end{array}$ & & & & -3.62 & -8.38 & 1.14 \\
\hline Proportion of patients benefiting & 22.56 & 19.78 & 25.34 & 0.45 & 0.40 & 0.51 \\
\hline Increased effectiveness & 10.74 & 8.71 & 12.77 & 0.43 & 0.35 & 0.52 \\
\hline Supportive services reduced & 10.84 & 9.23 & 12.44 & 17.46 & 14.70 & 20.21 \\
\hline Tax increase & 55.86 & 51.53 & 60.20 & & & \\
\hline Current model of care & & & & 4.05 & -0.72 & 8.82 \\
\hline
\end{tabular}

services. ${ }^{3,16}$ We used a DCE to evaluate how members of the UK public evaluated PMPs and the trade-offs necessary in their implementation.

We found that of the four vignette attributes, public support for PMPs was most contingent upon the financial cost. The cost to the taxpayer exerted a more significant influence than the combined influence of the other three attributes; in fact, the influence of the cost to the taxpayer, measured in this study by the CRAI, exerted a greater influence on how respondents valued PMPs than the combined influence of the other three attributes. The WTP analysis quantified the relationships between attributes, indicating that respondents would accept an approximate $£ 5$ increase in tax per capita for every 10-percentage-point increase in either the proportion of patients eligible for a PMP or the effectiveness of the PMP over $\mathrm{CMoC}$. The value attached to financial cost may represent a significant issue when PMPs include novel or expensive therapies. Ivacaftor, for example, which is available to the $5 \%$ of cystic fibrosis patients who possess a mutation in the CFTR gene, costs approximately $£ 182000$ per patient-year. ${ }^{9}$
We found that support for a PMP over $\mathrm{CMoC}$ increased as the proportion of patients eligible and the effectiveness of the treatment increased. By contrast, support decreased where PMP implementation required reductions in services from which all patients may benefit. Respondents indicated that they would be willing to pay almost $£ 17$ to avoid a reduction in such supportive services. This highlights an important caveat and chimes with findings reported from a survey of Canadian citizens: ${ }^{4}$ that PMP implementation must not come at the cost of other services. Policy makers may need to consider ring-fencing finances for $\mathrm{CMoC}$ should they consider implementing PMPs that are acceptable to the public.

Participants given vignettes relating to cystic fibrosis were more likely to support PMPs than those shown vignettes for depression. There may be several reasons for this disparity, some of which may be linked to disease-related characteristics. For example, another DCE found that respondents favoured redistribution of resources (linked with quality-adjusted life years) towards smaller groups of patients (such as those seen in PMPs), but particularly towards younger people, ${ }^{17}$ and we note that patients with cystic 
fibrosis are, on average, younger than those with depression. However, the same study observed that the public were less likely to support allocation of resources towards the treatment of diseases that significantly shorten life expectancy. Affective disorders are associated with premature mortality, ${ }^{18}$ but less than half of patients with cystic fibrosis live into their fifth decade. ${ }^{19}$ In any case, consideration of the different evaluative judgements being made for cystic fibrosis and depression in our DCE must take into account whether the respondents had equal knowledge of the prevalence and characteristics of the two conditions.

One clear difference between depression and cystic fibrosis, however, is the complexity of their respective aetiologies. Schildmann and Vollman recognise arguments that personalised medicine for some conditions may place obligations on patients to take greater responsibility for their health, but no such expectations can be placed on citizens living with diseases with causal genetic mechanisms such as cystic fibrosis. ${ }^{20}$ Thus, disorders and their consequences and treatments may be perceived entirely differently when viewed through the lens of personalised medicine. Respondents may link depression, a multifactorial disorder, with some sense of personal agency, and even self-stigma has been demonstrated to correlate negatively with support for allocating resources to psychiatric services. ${ }^{21}$ Alternatively, a comparative lack of support for PMPs in depression could reflect satisfaction with current treatment paradigms, rather than scepticism towards novel models. This appears unlikely when one considers the modest treatment effect of many antidepressant therapies ${ }^{22}$ and that access to psychological therapies in the UK is often regarded as suboptimal. ${ }^{23}$

Our previously discussed observation that respondents place importance on the preservation of existing services could be hypothesised to be driven by self-interest: 'if I developed depression, I wouldn't want lesser access to resources because a PMP necessitates more funding'. However, this hypothesis is difficult to reconcile with the finding that respondents were consistently more willing to fund a PMP for cystic fibrosis than one for depression, despite the much lower prevalence of cystic fibrosis. This suggests that the public's value system is not purely driven by utilitarianism or self-interest; a more complex value-based system may influence preferences regarding PMP implementation.

The size and representativeness of our study sample, and our use of a robust experimental design allowing quantification of respondents' preferences are important strengths of this study. Our findings are significant in demonstrating that condition-specific nuances to public support for PMPs exist and that bespoke approaches to implementation should be considered.

Our study did, however, have some limitations. First, we used only two conditions: cystic fibrosis and depression. These two conditions were chosen to represent an illness for which a PMP was in situ and one for which a PMP was more aspirational. However, the notable distinguishing characteristics of the two disorders, such as prevalence, mortality and aetiological complexity, precluded a more detailed analysis of the factors contributing to the differences in support for PMPs observed. Second, although we used a large representative sample and ensured that the vignettes were realistic, the respondents' decisions were hypothetical. It is possible that respondents' stated and actual choices could differ. Despite being hypothetical, several studies have shown that DCEs accurately reflect real-life decisions that respondents would make in similar circumstances. ${ }^{11}$ However, respondents' ranking of possible decisions are recognised to be subject to change when the methods employed change or the number of available alternatives increases (an observation known as 'preference reversal'). ${ }^{24}$ Although results from a method that presented vignettes in sets of three or more may have provided more data on the robustness of the decision-making involved, this increased complexity may have diminished respondents' understanding of the task or their willingness to complete it. Finally, although our survey used a representative sample of UK participants, the devolved powers across the UK mean that approach to healthcare can vary across the UK. This should be given careful consideration in any future policy regarding the adoption of personalised medicine approaches for the treatment of different diseases.

Mental health conditions are underrepresented in studies investigating the cost-effectiveness of personalised medicine ${ }^{25}$ and often fail to address how closely the financial cost of interventions is related to their public-perceived value. It will be necessary for further studies to explore how the public views mental health disorders and values the treatment and outcomes associated with them before meaningful comparisons with other disease groups can be made. Nevertheless, this study raises issues for the societal implementation of personalised medicine where only a subset of patients may benefit from targeted treatment. The general public may be unwilling to fund personalised medicine for few patients if this means a reduction in services from which all patients might benefit. Given the increased focus on using biomarker information to target treatments to specific subsets of patients, policy makers must address how personalised medicine treatments could potentially be funded.

Alan J. McMichael, PhD, Health \& Social Care Public Health Agency, Belfast, UK; Joseph P. M. Kane (D), PhD, Centre for Public Health, Queen's University Belfast, Belfast, UK: Jonathan J. Rolison, PhD, Department of Psychology, University of Essex, Essex, UK

Jonathan J. Rolison, PhD, Department of Psychology, University of Essex, Essex, UK;
Francis A. O'Neill, MD, Centre for Public Health, Queen's University Belfast, Belfast, UK; Marco Boeri, PhD, Health Preference Assessment, RTI Health Solutions, and School of Medicine, Dentistry and Biomedical Sciences, Queen's University Belfast, Belfast, UK; Frank Kee, MD, Centre for Public Health, and UKCRC Centre of Excellence for Public Health Research (NI), Queen's University Belfast, Belfast, UK

Correspondence: Joseph P.M. Kane. Email: joseph.kane@qub.ac.uk

First received 18 Aug 2021, final revision 13 Dec 2021, accepted 11 Jan 2022

\section{Data availability}

Data are available upon reasonable request to the corresponding author.

\section{Acknowledgements}

None.

\section{Author contributions}

A.M. contributed to the survey design and manuscript critique. J.K. contributed to the manuscript preparation and submission. J.R. contributed to the survey design and manuscript critique. T.O.N. contributed to the manuscript critique. M.B. contributed to the survey design, data analysis and manuscript preparation. F.K. contributed to the survey design and manuscript critique. A.M. and J.K. made equal contributions to the project. M.B. and F.K. made equal contributions to the project.

\section{Funding}

Financial support for this study was provided by a grant from the Department of Education. The funding agreement ensured the authors' independence in designing the study, interpreting the data, and writing and publishing the report.

\section{Declaration of interest}

None.

\section{References}

1 Hall IP. Stratified medicine: drugs meet genetics. Eur Respir Rev 2013; 22(127): 53-7.

2 Jukic MM, Smith RL, Haslemo T, Molden E, Ingelman-Sundberg M. Effect of CYP2D6 genotype on exposure and efficacy of risperidone and aripiprazole: a retrospective, cohort study. Lancet Psychiatry 2019; 6(5): 418-26. 
3 Trusheim MR, Berndt ER. Economic challenges and possible policy actions to advance stratified medicine. Per Med 2012; 9(4): 413-27.

4 Bombard Y, Abelson J, Simeonov D, Gauvin F. Citizens ' perspectives on personalized medicine : a qualitative public deliberation study. Eur J Hum Genet 2013; 21(11): 1197-201.

5 Department of Health. No Health Without Mental Health: A Cross-Government Mental Health Outcomes Strategy for People of All Ages. 2011 (https://www. gov.uk/government/publications/no-health-without-mental-health-a-cross-government-outcomes-strategy [cited 1 Aug 2021]).

6 Fineberg NA, Haddad PM, Carpenter L, Gannon B, Sharpe R, Young AH, et al. The size, burden and cost of disorders of the brain in the UK J Psychopharmacol 2013; 27(9): 761-70.

7 Johnson FR, Lancsar E, Marshall D, Kilambi V, Mühlbacher A, Regier DA, et al. Constructing experimental designs for discrete-choice experiments: report of the ISPOR conjoint analysis experimental design good research practice task force. Value Heal 2013; 16(1): 3-13.

8 Hauber AB, González JM, Groothuis-Oudshoorn CGM, Prior T, Marshall DA Cunningham $\mathrm{C}$, et al. Statistical methods for the analysis of discrete choice experiments: a report of the ISPOR conjoint analysis good research practices task force. Value Heal 2016; 19(4): 300-15.

9 Wainwright CE, Elborn JS, Ramsey BW, Marigowda G, Huang X, Cipolli M, et al. Lumacaftor-ivacaftor in patients with cystic fibrosis homozygous for Phe508del CFTR. N Engl J Med 2015; 373(3): 220-31.

10 Hauber AB, Arden NK, Mohamed AF, Johnson FR, Peloso PM, Watson DJ, et al. A discrete-choice experiment of United Kingdom patients' willingness to risk adverse events for improved function and pain control in osteoarthritis. Osteoarthritis Cartilage 2013; 21(2): 289-97.

11 Salampessy BH, Veldwijk J, Jantine Schuit A, van den Brekel-Dijkstra K, Neslo REJ, Ardine de Wit G, et al. The predictive value of discrete choice experiments in public health: an exploratory application. Patient 2015; 8(6): 521-9.

12 Train KE. Discrete Choice Methods with Simulation (2nd edn). Cambridge University Press, 2009.

13 Hess S, Train K. Correlation and scale in mixed logit models. J Choice Model 2017; 23: 1-8

14 Hole AR. Fitting mixed logit models by using maximum simulated likelihood Stata J 2007: 7: 388-401.

15 Office for National Statistics. National Records of Scotland, Northern Ireland Statistics and Research Agency, 2011 Census Aggregate Data.
UK Data Service, 2017 (https://discover.ukdataservice.ac.uk/doi/2011censusaggregate [cited 1 Aug 2021]).

16 Balfour-Lynn IM. Personalised medicine in cystic fibrosis is unaffordable. Paediatr Respir Rev 2014; 15: 2-5.

17 Skedgel C, Wailoo A, Akehurst R. Societal preferences for distributive justice in the allocation of health care resources: a latent class discrete choice experiment. Med Decis Making 2015; 35(1): 94-105.

18 Plana-Ripoll O, Pedersen CB, Agerbo E, Holtz Y, Erlangsen A, Canudas-Romo V et al. A comprehensive analysis of mortality-related health metrics associated with mental disorders: a nationwide, register-based cohort study. Lancet 2019; 394(10211): 1827-35.

19 Keogh RH, Szczesniak R, Taylor-Robinson D, Bilton D. Up-to-date and projected estimates of survival for people with cystic fibrosis using baseline characteristics: a longitudinal study using UK patient registry data. J Cyst Fibros 2018; 17(2): 218-27.

20 Schildmann J, Vollmann J. Personalized medicine: conceptual, ethical, and empirical challenges. In Handbook of the Philosophy of Medicine (eds T Schramme, S Edwards): 1-11. Springer, 2017.

21 DeLuca JS, Vaccaro J, Seda J, Yanos PT. Political attitudes as predictors of the multiple dimensions of mental health stigma. Int J Psychiatry 2018; 64(5): 459-69.

22 Cipriani A, Furukawa TA, Salanti G, Chaimani A, Atkinson LZ, Ogawa $Y$, et al. Comparative efficacy and acceptability of 21 antidepressant drugs for the acute treatment of adults with major depressive disorder: a systematic review and network meta-analysis. Lancet 2018; 391(10128): 1357-66.

23 Clark DM, Canvin L, Green J, Layard R, Pilling S, Janecka M. Transparency about the outcomes of mental health services (IAPT approach): an analysis of public data. Lancet 2018; 391(10121): 679-86.

24 Oliver AJ, Sorenson C. Importance of preference reversals in the valuation of health and healthcare. Expert Rev Pharmacoecon Outcomes Res 2008; 8(2): 95-9.

25 Kasztura M, Richard A, Bempong NE, Loncar D, Flahault A. Cost-effectiveness of precision medicine: a scoping review. Int J Public Health 2019; 64(9): 1261-71. 